\title{
Clinical Features of Severe Tsutsugamushi Disease Complicated with Hemophagocytic Lymphohistiocytosis
}

\author{
Linglong Lu1*, Hainan Xu², Dongping Huang3, Shumei Peng4 \\ ${ }^{1}$ Department of Pediatric Immunization, Guangdong Maternal and Child Health Hospital, Guangzhou 510500, China \\ ${ }^{2}$ Department of Pediatric Respiratory Diseases, Guangdong Maternal and Child Health Hospital, Guangzhou 510500, \\ China \\ ${ }^{3}$ Department of Pediatric Infection, Guangdong Maternal and Child Health Hospital, Guangzhou 510500, China \\ ${ }^{4}$ Department of Pediatric Intensive Care, Guangdong Maternal and Child Health Hospital, Guangzhou 510500, China \\ *Corresponding author: Linglong Lu, $3051309461 @$ qq.com
}

\begin{abstract}
The article discusses a case of severe tsutsugamushi disease complicated with hemophagocytic syndrome in Guangdong Maternal and Child Health Hospital and concludes that blood purification technology has significant therapeutic effect among children with severe HLH complicated with multiple organ dysfunction.
\end{abstract}

Keywords: Tsutsugamushi disease; Hemophagocytic lymphohistiocytosis

Publication date: September 2021; Online publication: September 30, 2021

\section{Introduction}

Tsutsugamushi disease, also known as scrub typhus, is an acute infectious disease caused by an obligate intracellular bacterium, Rickettsia tsutsugamushi ${ }^{[1,2]}$, which is parasite to chigger mites. Over the years, tsutsugamushi disease has become a serious health problem, affecting 1 million people every year with 1 billion people being vulnerable to this infection. Although it is serious and spreads in southern and eastern Asia as well as the Pacific Rim, it is a health issue that has been neglected for a long time ${ }^{[3]}$. The onset of the disease is sudden, and its clinical manifestations are diverse and complex, including fever, headache, vomiting, toxemia, rash, eschar, and lymphadenopathy. If treated in time, the cure rate is high but with a few sequelae. Delayed diagnosis and treatment can lead to serious complications, including disseminated intravascular coagulation (DIC), acute respiratory distress syndrome (ARDS), and hemophagocytic lymphohistiocytosis (HLH) ${ }^{[4]}$. According to the latest statistics, in the absence of treatment for tsutsugamushi disease, the average mortality of patients can be as high as $70 \%{ }^{[5]}$. Therefore, timely diagnosis and accurate treatment of tsutsugamushi disease should be emphasized. Hemophagocytic lymphohistiocytosis (HLH), also known as hemophagocytic syndrome, is a rare and highly fatal disease ${ }^{[6]}$, which can be divided into primary hemophagocytic lymphohistiocytosis (PHLH) and secondary hemophagocytic lymphohistiocytosis (SHLH) according to the cause. PHLH involves autosomal or Xlinked recessive inheritance with related gene abnormalities, whereas SHLH is related to a variety of stimuli, such as infections, malignant tumors, and conditions characterized by immunosuppression; however, the incidence of tsutsugamushi disease complicated with hemophagocytic syndrome is extremely low ${ }^{[7]}$. Agrwal once reported a case of tsutsugamushi disease complicated with hemophagocytic syndrome along with serious complications of the nervous system. Its treatment is mainly pathogen-directed antibiotic 
treatment without the routine use of immunomodulator therapy ${ }^{[8]}$. The following is a discussion on a case of severe tsutsugamushi disease complicated with hemophagocytic syndrome in Guangdong Maternal and Child Health Hospital.

\section{Case introduction}

The patient is SM, a 6-year-old male student. Due to fever and abdominal pain for more than 5 days and cough for more than 4 days, he was admitted on April 5, 2021. The physical examination on admission was documented: mental fatigue, swollen eyelids and face, no chapped lips, pharyngeal congestion, no strawberry tongue, and ecchymosis on the neck. No enlarged lymph nodes were palpable. His breathing was smooth, respiratory sounds from both his lungs were thick but reduced over the right lower lung, and dry rales could be heard from both lungs. His heart sounds were strong and regular without any pathological murmur heard in each valve. Abdominal distension was noted, his liver was palpable $4 \mathrm{~cm}$ below the right rib, having sharp edge, tender, and not extending toward to left ribs. Tenderness was felt at the right lower quadrant of the abdomen. No shifting dullness elicited. Eschar could be seen on the inner side of his left thigh (Figure 1). Pin-sized petechiae were seen scattered over his limbs. Both lower limbs had pitting edema. Meningeal irritation signs and pathological signs of the nervous system were not elicited.

Prior to admission, the results of the investigations that were carried out on April 3, 2021, are as follows: plain chest and abdominal CT (computed tomography) scan showed slight pleural effusion on the right (about $7 \mathrm{~mm}$ deep), splenomegaly, as well as abdominal and pelvic effusion; routine blood and C-reactive protein test showed leukocyte count was $11.92 \times 10^{9} / \mathrm{L}$, C-reactive protein was $117.88 \mathrm{mg} / \mathrm{l}$, neutrophil percentage was $86.8 \%$, lymphocyte percentage was $7.7 \%$, platelet count was $23 \times 10^{9} / \mathrm{L}$, red blood cell count was $4.81 \times 10^{12} / \mathrm{L}$, and hemoglobin was $123 \mathrm{~g} / \mathrm{L}$; procalcitonin was $2.60 \mathrm{ng} / \mathrm{ml}$; for blood biochemistry, no abnormalities were found in the renal function, ions, and myocardial enzymes; however, alanine aminotransferase was $239.85 \mathrm{U} / \mathrm{L}$, total bilirubin was $51.3 \mathrm{umol} / \mathrm{L}$, direct bilirubin was $37.0 \mathrm{umol} / \mathrm{L}$, and indirect bilirubin was $14.30 \mathrm{umol} / \mathrm{L}$; five immunological tests were carried out, where immunoglobulin $\mathrm{G}$ was $4.62 \mathrm{~g} / \mathrm{L}(\downarrow)$ and complement $\mathrm{C} 3$ was $0.70 \mathrm{~g} / \mathrm{L}(\downarrow)$ without abnormalities found in the rest.

After admission, investigations were also carried out. On April 5, 2021, ultrasound scan showed bilateral pleural effusion, but there was no effusion found in the abdominal cavity. Plain abdominal CT scan showed: (1) inflammatory changes in both lungs, mainly interstitial lesions, and bilateral pleural effusion; (2) at the right lower quadrant of the abdomen at the appendix area, no appendicitis was seen, please correlate clinically; (3) a small amount of peritoneal effusion; (4) large liver, hepatic lymphangitis, and splenomegaly. Echocardiography showed that his cardiac structure and left ventricular systolic function were normal. His procalcitonin level was $2.00 \mathrm{ng} / \mathrm{ml}$. Routine blood tests showed that his hemoglobin $(\mathrm{Hgb})$ was $97 \mathrm{~g} / \mathrm{L}(\downarrow)$, platelet count (PLT) was $13 \times 10^{9} / \mathrm{L}$, white blood cell count (WBC) was $7.97 \times 10^{9} / \mathrm{L}$, neutrophil percentage was $66.9 \%$, and lymphocyte percentage was $16.1 \%$. In the detection of eight respiratory pathogens, human parainfluenza virus (PIV) IgM was positive (+) without any abnormalities found in the rest. Blood biochemistry showed that triglyceride (TG) was $4.56 \mathrm{mmol} / \mathrm{L}$, alanine aminotransferase (ALT) was $219 \mathrm{u} / \mathrm{L}$, aspartate aminotransferase was $256 \mathrm{u} / \mathrm{L}$, total protein (TP) was 45.3 $\mathrm{g} / \mathrm{L}$, albumin (ALB) was $23.8 \mathrm{~g} / \mathrm{L}$, globulin (GLO) was $21.5 \mathrm{~g} / \mathrm{L}$, and albumin-to-globulin ration (A/G) was 1.1. No abnormalities were found in his bilirubin levels, renal function, ions, and myocardial enzymes. However, his serum ferritin was $3308.71 \mathrm{ng} / \mathrm{ml}(\uparrow)$. In regard to the six parameters of coagulation, his partially activated thrombin time (APTT) was 29.7 seconds, thrombin time (TT) was 23.2 seconds ( $\uparrow$ ), prothrombin time (PT) was 11.7 seconds, and fibrinogen (FBG) was $0.81 \mathrm{~g} / \mathrm{L}(\downarrow)$ ). On April 6, Weil Felix reaction showed that serum OXK titer was 1:320 (+). On April 7, 2021, cerebrospinal fluid test revealed that mononuclear cells were $8 \times 10^{6} / \mathrm{L}$, total cells were $15 \times 10^{6} / \mathrm{L}$, blood was positive (+), protein was 543 $\mathrm{mg} / \mathrm{L}(\uparrow)$. The complete lymphocyte subset panel analysis (TBNK) showed that total T lymphocyte 
percentage was $80.97 \%$, helper/induced $\mathrm{T}$ cell percentage (CD4 + T\%) was $23.33 \%(\downarrow)$, inhibitory/cytotoxic T lymphocyte percentage (CD8 + T\%) was 56.23\% ( $\uparrow$ ), helper T lymphocyteinhibitory T lymphocyte ratio (CD4 + T/CD8 + T) was $0.41(\downarrow)$, natural killer $(\mathrm{NK})$ cell percentage was $8.35 \%(\downarrow)$, B lymphocyte was $7.17 \%$, total T lymphocyte count was 1153.27 PCS/UL, B lymphocyte count was 102.14 PCS/UL ( $\downarrow$ ), NK cell count was 118.97 PCS/UL, helper/induced T cell count (CD4 + T\#) was 332.23 PCS/UL $(\downarrow)$, and inhibitory/cytotoxic T lymphocyte count (CD8 + T\#) was 800.83 PCS/UL. Bone marrow cytology (Figure 2) revealed that more phagocytic histiocytes can be seen, and a bone marrow picture of hemophagocytic syndrome was considered with clinical correlation. The detection of pathogens in the peripheral blood and cerebrospinal fluid by metagenomic sequencing revealed the presence of Orientia tsutsugamushi. There were no obvious abnormalities in PCR-EBV (Ebstein-Barr virus by polymerase chain reaction) detection, PCR-CMV (cytomegalovirus) detection, fat Das reaction, antineutrophil cytoplasmic antibody test, three parameters of anticardiolipin antibodies, two parameters for rheumatism, hepatitis $\mathrm{C}(\mathrm{HCV})$ antibody determination, hepatitis B two half and half, NS1 antigen detection for dengue, Treponema pallidum particle agglutination (TPPA) test, human immunodeficiency virus (HIV) antibody determination, four parameters for blood lipid, and routine stool examination.

In consideration of the obvious systemic inflammatory reaction and sepsis after admission, the child was given imipenem-cilastatin and azithromycin for anti-infection. On the second day after admission (the seventh day of the total course of the disease), the child developed dyspnea and persistent high fever. Endotracheal intubation was done, and he was started on ventilator-assisted ventilation - synchronized intermittent mandatory ventilation (SIMV) for a week, followed by plasma exchange (once a day, $\mathrm{X}^{2}$ days) and continuous hemofiltration (113 hours). After the induction of methylprednisolone, he was gradually tapered to oral prednisone tablets (the total course of treatment was about 8 weeks), supplemented with gamma globulin, albumin to correct hypoalbuminemia, cryoprecipitate and fresh frozen plasma to correct coagulation disorders, mannitol and furosemide to reduce intracranial pressure, omeprazole to inhibit acid and protect the stomach, glutathione to protect the liver, isoproterenol and dobutamine to improve circulation, etc. He was hospitalized for 17 days.

The final diagnoses were as follows: (1) hemophagocytic lymphohistiocytosis; (2) sepsis; (3) severe tsutsugamushi disease; (4) rickettsial encephalitis; (5) multiple organ dysfunction syndrome (liver, lung, blood, brain, and heart); (6) acute respiratory distress syndrome; (7) severe pneumonia; (8) acute appendicitis; (9) multiple serous effusion (pleural effusion and peritoneal effusion); (10) hypoproteinemia; (11) moderate anemia; (12) mitral regurgitation; (13) tricuspid regurgitation; (14) cholestasis; (15) gastrointestinal bleed; (16) rhinitis; (17) epistaxis. The child was followed-up regularly after discharge. His routine blood, blood biochemistry, immune function, and other indicators were basically normal. 


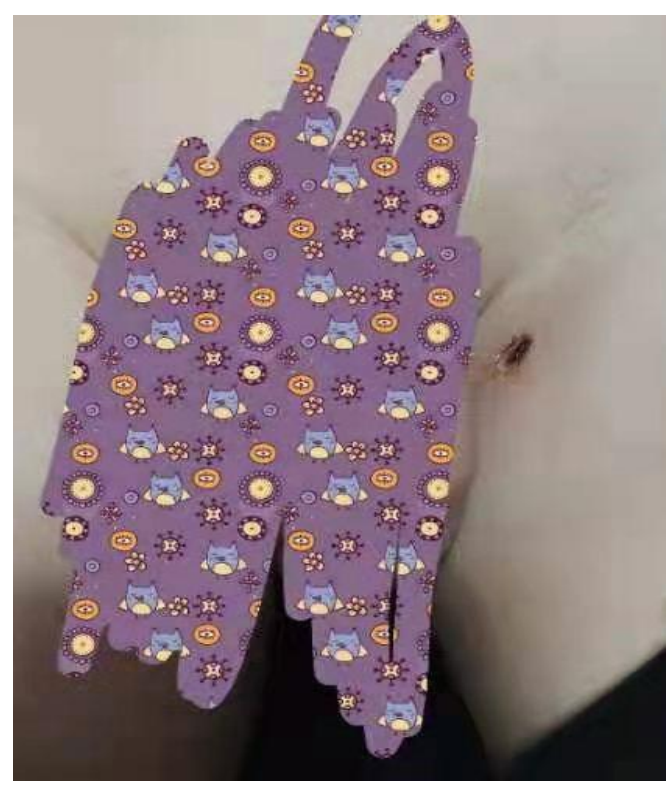

Figure 1. Eschar at the inner left thigh

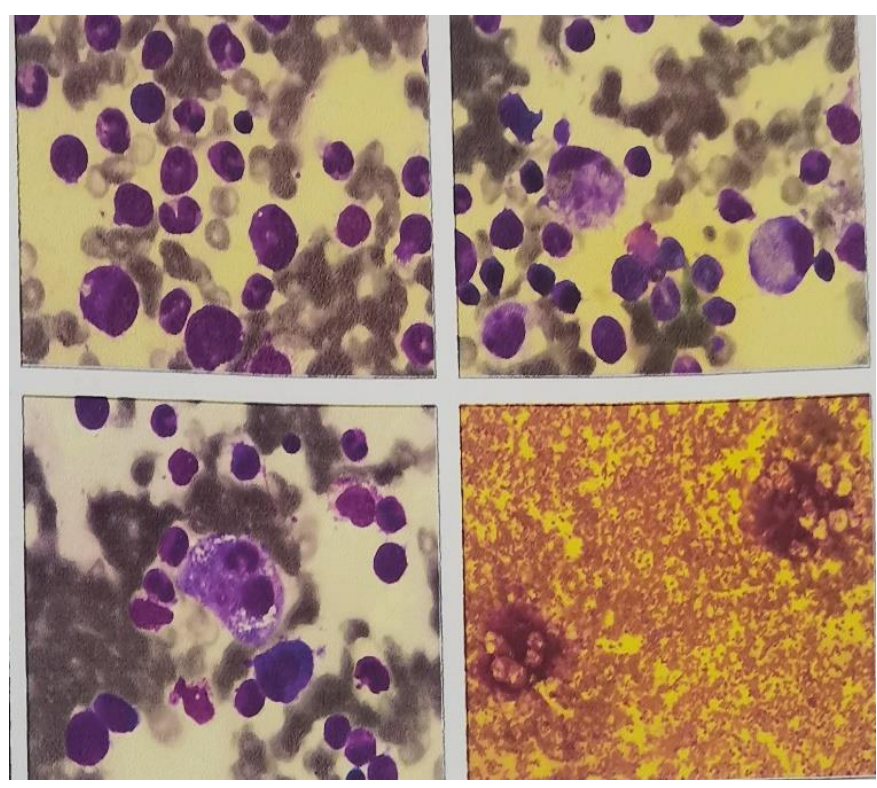

Figure 2. Bone marrow cytology

\section{Discussion}

Tsutsugamushi disease, also known as scrub typhus, is a tropical disease transmitted through the bite of Chiggers that feed on infected rodents as the main source of infection. The disease is generally self-limited one week after its onset, but in a few cases, serious complications or even death can occur ${ }^{[8,10]}$. The main endemic regions of the disease are in the tsutsugamushi triangle, which starts from the northernmost tip of Korea, Japan, and Russia in the north, reaching northern Australia in the south, and extending to Pakistan as well as Afghanistan in the West ${ }^{[8]}$. In regard to this, China is also included as an important endemic area ${ }^{[11]}$. Farmers, jungle workers, and children are the main population affected by the disease ${ }^{[12]}$. The acute fever caused by the disease is often confused with other fever of unknown origin (FUO), such as typhoid fever, leptospirosis, dengue fever, malaria, and viral hemorrhagic fever. It is one of the important causes of FUO in endemic areas ${ }^{[13]}$.

Tsutsugamushi disease is a systemic disease. The typical early clinical manifestations are fever, eschar, rash, and other general symptoms. Some patients also have abdominal pain, especially among children ${ }^{[14]}$. In the case that was discussed earlier, the child had abdominal pain complicated with acute appendicitis. There is a need to pay attention to clinical differentiation. Severe cases in children may further lead to respiratory and circulatory failure, encephalitis, as well as life threatening complications, such as multiple organ dysfunction ${ }^{[15,16]}$. Severe tsutsugamushi disease ${ }^{[17]}$ refers to one of the following: complicated with interstitial pneumonia or dyspnea; renal failure; meningoencephalitis with altered consciousness; shock; myocarditis; gastrointestinal bleed; death. Serum OXK test more than 1:160 and isolated pathogens have higher values in diagnosing the disease ${ }^{[18]}$. Eschar is of great value in the clinical diagnosis and treatment of the disease. A typical eschar from the bite is made up of a black eschar in the center with red halo around it; this can be seen in the picture from the case (Figure 1). In the case that was discussed, the child lives in Guangdong. He had a history of mountain and grassland activities three days before the onset, persistent fever for more than 5 days, and an obvious eschar on the inner side of his groin (Figure 1). Weil Felix reaction showed that serum OXK was 1:320 (+) and the detection of pathogens in peripheral blood and cerebrospinal fluid by metagenomics sequencing revealed the presence of Orientia tsutsugamushi. During the course of the disease, there were hemophagocytic syndrome with pulmonary interstitial inflammation 
complicated with respiratory distress syndrome, change in consciousness caused by rickettsial encephalitis, thrombocytopenia, anemia, and other abnormal blood changes. In addition, his liver function was damaged, and he had hypoproteinemia, gastrointestinal bleed, multiple serous effusion, and other serious complications. All these are consistent with the diagnosis of severe tsutsugamushi disease.

Rickettsia tsutsugamushi is an obligate intracellular parasitic microorganism, which is widely distributed in vascular endothelial cells and macrophages. Many immunologists believe that it induces the activation of $\mathrm{T}$ lymphocytes and the preferential differentiation of Th1 cells through the processing and presentation of its antigen ${ }^{[19]}$. Rickettsia tsutsugamushi is associated with hypercytokinemia, and it increases macrophage colony stimulating factor (M-CSF), interferon (IFN), as well as interleukin-10 (IL10) significantly. IFN can activate macrophages, which is similar to IL-10 chemotactic response, enhancement of $\mathrm{CD}^{+} \mathrm{T}$ lymphocyte cytotoxicity, and overactivation of THL cells. M-CSF can also stimulate the proliferation and enhance the phagocytosis of macrophages, proliferate and activate a large number of $\mathrm{CD}^{+} \mathrm{T}$ lymphocytes, clear intracellular rickettsial infection, and induce the phagocytosis of blood products. There have been reports on tsutsugamushi disease that is complicated with hemophagocytic lymphohistiocytosis. Recent studies ${ }^{[8]}$ have shown that age less than 7 years old, prolong fever, convulsion, acute respiratory distress syndrome (ARDS), and multiple organ dysfunction syndrome (MODS) are high risk factors for tsutsugamushi disease complicated with hemophagocytic lymphohistiocytosis among children ${ }^{[20,21]}$. The treatment is mainly pathogen-directed antibiotic treatment without a routine use of immunomodulator therapy. In the case that has been discussed, the child was 6 years of age and had fever for more than 5 days. There was also a quick development of acute respiratory distress syndrome. More phagocytic histiocytes were seen in the bone marrow cytology (Figure 2). Serological examination and TBNK assay showed that $\mathrm{CD} 4^{+} \mathrm{T}$ cells and NK cells decreased while $\mathrm{CD} 8^{+} \mathrm{T}$ cells increased, resulting in immune disorders, cytokine storm, and coagulation disorders. Anemia, thrombocytopenia, and other blood product phagocytosis phenomena along with the obvious decrease of fibrinogen were consistent with a series of systemic immune and inflammatory reactions after tsutsugamushi infection.

The treatment of tsutsugamushi disease mainly focuses on the pathogen and organ support. Early effective antibiotics (doxycycline, macrolides, quinolones, and chloramphenicol) treatment are provided, where children are usually treated with azithromycin with a general course of 7-10 days. In this case, he was given azithromycin and after 10 days of treatment, the pathogen detection returned to negative. However, this case had serious complications, including respiratory failure, rickettsial encephalitis, hemophagocytic syndrome, liver function damage, multiple serous cavity effusion, gastrointestinal bleed, acute appendicitis, and other multiple organ dysfunctions. Moreover, the hospital stay was long. Although the child had to go through all these, there were no sequelae, and the prognosis was good. The key to consider was the early and timely use of plasma exchange and continuous hemofiltration technology to remove pathogens and inflammatory factors. At the same time, immunosuppressive therapy was also used to prevent further deterioration of the disease and save the life of child in time. Oral prednisone was also given at a later stage. All indicators of his follow-up were good, and the drug was then tapered. The treatment used in this case was inconsistent with the chemotherapy regimen advocated by the guidelines for HLH diagnosis and treatment (including the use of glucocorticoids, cyclosporine, and etoposide).

Hemophagocytic lymphohistiocytosis (HLH) is a clinical syndrome characterized by pathological inflammatory response caused by the extreme activation of immune responses. Evidence have shown that infection associated hemophagocytic lymphohistiocytosis (IAHS) is induced by infectious factors due to specific immune stimulation in patients with genetic susceptibility. The latest research progress about IAHS ${ }^{[22]}$ suggested that the pathogenesis of IAHS is complex. Its clinical manifestations include fever, 
enlargement of liver, spleen, and lymph nodes, as well as critical conditions, such as abnormal liver function, multiple serous cavity effusion, and multiple organ dysfunction in some patients. There is high early mortality with his syndrome as reported by Zhang Yucai ${ }^{[23]}$. It is necessary to attach great importance to the early identification and treatment of hemophagocytic lymphohistiocytosis among children in intensive care units. In terms of treatment, the best treatment should be determined according to the pathological basis of each patient that leads to this pathological inflammatory reaction ${ }^{[24]}$, including anti-infectives, adjusting the body's immune level according to the disease progress, transplantation, symptomatic support treatment, and removing external stimulating factors. Therefore, early diagnosis, removing potential etiology, and individualized treatment are the key factors to improve the prognosis of HLH. With in-depth understanding of the disease and the progress of treatment methods, many more blood purification technologies ${ }^{[8]}$ have been applied in the treatment of hemophagocytic lymphohistiocytosis. Blood purification technology plays a very important role in clearing the early cytokine storm, improving the survival rate, and shortening hospital stay. Tao Xiaofen and other researchers ${ }^{[25,26]}$ had recently reported the use of plasma exchange in hemophagocytic lymphohistiocytosis. They concluded that blood purification technology has significant therapeutic effect in children with severe HLH complicated with multiple organ dysfunction and the mortality decreases significantly within 7 days and after 2 months. Our team has also introduced a large number of blood purification technologies in the treatment of hemophagocytic lymphohistiocytosis, playing a great role in reducing early mortality with optimistic outcomes. Clinical data and other related research are still further improving in regard to this specific research results.

\section{Disclosure statement}

The authors declare that there is no conflict of interest.

\section{References}

[1] Amano K, Tamura A, Ohashi N, et al., 2987, Deficiency of Peptidoglycan and Lipopolysaccharide Components in Rickettsia Tsutsugamushi. Infect Immun, 55: 2290-2292.

[2] Paris DH, Shelite TR, Day NP, et al., 2013, Unresolved Problems Related to Scrub Typhus: A Seriously Neglected Life-Threatening Disease. Am J Trop Med Hyg, 89: 301-307. 10.4269/ajtmh.13-0064

[3] Xu G, Walker DH, Jupiter D, et al., 2017, A Review of the Global Epidemiology of Scrub Typhus. PLoS Negl Trop Dis, 11(11): e0006062. 10.1371/journal.pntd.0006062

[4] Cascio A, Correnti P, Iaria C, 2013, Scrub Typhus, Acute Respiratory Distress, and Hemophagocytic Lymphohistiocytosis. Int J Infect Dis, 17: e662. 10.1016/j.ijid.2013.03.006

[5] Bonell A, Lubell PN, Newton JA, et al., 2017, Estimating the Burden of Scrub Typhus: A Systematic Review. PLoS Negl Trop Dis, 11: e0005838. 10.1371/journal.pntd.0005838

[6] Usmani GN, Woda BA, Newburger PE, 2013, Advances in Understanding the Pathogenesis of HLH. Br J Haematol, 161: 609-622.

[7] Agrwal S, Dabas A, Mantan M, et al., 2019, Hemophagocytic Lymphohistiocytosis with Neurological Manifestations in an Infant with Scrub Typhus: A Rare Fatal Occurrence. Trop Doct, 49(1): 52-53. $10.1177 / 0049475518804696$

[8] Basu A, Chowdhoury SR, Sarkar M, et al., 2021, Scrub Typhus-Associated Hemophagocytic Lymphohistiocytosis. J Trop Pediatr, 67(1): 1-10. 10.1093/tropej/fmab001 
[9] Paris DH, Shelite TR, Day NP, et al., 2013, Unresolved Problems Related to Scrub Typhus: A Seriously Neglected Life-Threatening Disease. Am J Trop Med Hyg, 89(2): 301-307. 10.4269/ajtmh.13-0064

[10] Park JW, Kim SH, Park DW, et al., 2018, Molecular Epidemiology of an Orientia Tsutsugamushi Gene Encoding a 56-Kda Type-Specific Antigen in Chiggers, Small Mammals, and Patients from the Southwest Region of Korea. Am J Trop Med Hyg, 98(2): 616-624. 10.4269/ajtmh.17-0070

[11] Qi Y, Yin Q, Shao Y, et al., 2018, Development of a Rapid and Visual Nucleotide Detection Method for A Chinese Epidemic Strain of Orientia Tsutsugamushi Based on Recombinase Polymerase Amplification Assay and Lateral Flow Test. Int J Infect Dis, 70: 42-50. 10.1016/j.ijid.2018.03.003

[12] Yue Y, Wang Y, Li G, et al., 2020, Epidemiological Characteristics of Tsutsugamushi Disease in Chinese Mainland from 2006 to 2018. Surveillance, 35(4): 301-306. 10.3784/j.issn.10039961.2020.04.007

[13] Gautam R, Parajuli K, Tshokey T, et al., 2020, Diagnostic Evaluation of IgM ELISA and IgM Immunofluorescence Assay for the Diagnosis of Acute Scrub Typhus in Central Nepal. BMC Infect Dis, 20(1): 138. 10.1186/s12879-020-4861-y

[14] Valbuena G, Walker DH, 2013, Approaches to Vaccines Against Orientia Tsutsugamushi. Cell Infect Microbiol, 2: 1-18. 10.3389/fcimb.2012.00170

[15] Yasunaga H, Horiguchi H, Kuwabara K, et al., 2011, Delay in Tetracycline Treatment Increases the Risk of Complications in Tsutsugamushi Disease: Data from the Japanese Diagnosis Procedure Combination Database. Intern Med, 50(1): 37-42. 10.2169/internalmedicine.50.4220

[16] Koh GC, Maude RJ, Paris DH, et al., 2010, Diagnosis of Scrub Typhus. Am J Trop Med Hyg, 82(3): 368-370. 10.4269/ajtmh.2010.09-0233

[17] Wang RD, Dai Y, Gong Y, 2021, Progress in Diagnosis and Treatment of Severe Tsutsugamushi Disease. Chinese Journal of Medicine, 2(28): 0376-2491.

[18] Feng X, Jia X, Ruan Y, Guo L, et al., 2020, Research Progress of Laboratory Diagnosis Methods of Tsutsugamushi Disease. Chinese Journal of Laboratory Medicine, 43(12): 1248-1251.

[19] Evans SM, Rodino KG, Adcox HE, et al., 2018, Orientia Tsutsugamushi Uses Two Ank Effectors to Modulate NF-Kb P65 Nuclear Transport and Inhibit NF-Kb Transcriptional Activation. PLoS Pathog, 14(5): e1007023. 10.1371/journal.ppat.1007023

[20] Canna SW, Marsh RA, 2020, Pediatric Hemophagocytic Lymphohistiocytosis. Blood, 135(16): 13321343. 10.1182/blood.2019000936

[21] Jun GL, Jin Y, et al., 2014, Analysis of Clinical Characteristics of 19 Cases of Hemophagocytic Syndrome Secondary to Tsutsugamushi Disease. Chinese Journal of Pediatrics.

[22] Wang Y, Zhang L, 2019, Progress in Diagnosis and Treatment of Infection Related Hemophagocytic Syndrome. World Clinical Drugs.

[23] Zhang Y, Cui Y, et al., 2013, Pay Attention to the Diagnosis and Treatment of Critical Hemophagocytic Syndrome in Children's Intensive Care Unit, 5(2): 1671-0282.

[24] Allen CE, Mc Clain KL, Wang Z, 2015, Pathophysiology and epidemiology of hemophagocytic syndrome. Hematology Am Soc Hematol Educ Program, 2015: 177-82.

[25] Tao X, 2015, Plasma Exchange Combined with Chemotherapy in the Treatment of a Child with Hemophagocytic Syndrome and Literature Review. World Latest Medicine Information. 15(55).

[26] Zhang Y, Xiao Z, 2018, Effect of Plasma Exchange Combined with Continuous Blood Purification on Pediatric Hemophagocytic Syndrome. Journal of Practical Shock, 2(4): 223-228. 